\title{
Refractory Endometrial Endometrioid Adenocarcinoma
}

National Cancer Institute

\section{Source}

National Cancer Institute. Refractory Endometrial Endometrioid Adenocarcinoma. NCI Thesaurus. Code C150098.

Endometrial endometrioid adenocarcinoma that is resistant to treatment. 